MS41-02

\section{Experiences developing and publishing a medium-sized software project: CrystFEL}

Thomas White ${ }^{1}$

1. Center for Free-Electron Laser Science, Deutsches ElektronenSynchrotron DESY, Hamburg, Germany

email: taw@physics.org

CrystFEL [1,2] is a free and open-source suite of programs for processing data from "serial crystallography" experiments, starting from detector frames and ending with merged intensities ready to be imported into other packages such as CCP4 and PHENIX. As of June 2018, it has been in development for $8 \frac{1}{2}$ years and publicly available for just over 6 years. About 100 journal articles involved its use and 25 people from 8 different institutions have contributed code, with many more people contributing useful ideas and feedback.

This talk will describe our experiences developing CrystFEL and making it available to crystallographers around the world. How can the complexities of scientific data processing and high-performance computing environments be managed for the best overall results? What is the best way to get new users started, or to make changes to the software without tripping up the more experienced? And how can we encourage users who have programming skills to become contributors?

References:

[1] White, T. A. et al. (2016). J. Appl. Cryst. 49, 680-689.

[2] https://www.desy.de/ twhite/crystfel/

Keywords: serial crystallography, CrystFEL, XFEL
MS41-03

\section{Using Mathematica ${ }^{\circledR}$ as a platform for crystallographic computing}

Stian Ramsnes ${ }^{1}$, Helge Bøvik Larsen ${ }^{1}$, Gunnar Thorkildsen ${ }^{1}$

1. Department of Mathematics and Physics, University of Stavanger, Stavanger, Norway

email: stian.p.ramsnes@uis.no

A comprehensive Mathematica ${ }^{\circledR}$ [1] package for crystallographic computations has been developed.

Complete representations of space groups (point groups) and cell transformations based on International Tables for Crystallography, volume A [2] together with scattering factors from International Tables for Crystallography, volume $\mathrm{C}$ [2] and cross sections from xraylib [3] are built into the package.

Featured functionalities include calculation of structure factors, linear absorption coefficients and various crystallographic transformations.

The crystal data used by the package is normally generated from external *.cif files.

The package comes with a dynamic documentation seamlessly integrated with the Mathematica system, including code, examples and options.

From the onset, minimal Mathematica experience is needed to make use of the package.

It may be a helpful supplement in research and teaching where crystallography and X-ray diffraction is essential.

Although Mathematica is a proprietary software, all the code of the package is open source.

It may easily be extended to cover user-specific applications.

A comprehensive Mathematica ${ }^{\circledR}[1]$ package for crystallographic computations has been developed.

Complete representations of space groups (point groups) and cell transformations based on International Tables for Crystallography, volume A [2] together with scattering factors from International Tables for Crystallography, volume $\mathrm{C}$ [2] and cross sections from xraylib [3] are built into the package.

Featured functionalities include calculation of structure factors, linear absorption coefficients and various crystallographic transformations.

The crystal data used by the package is normally generated from external *.cif files.

The package comes with a dynamic documentation seamlessly integrated with the Mathematica system, including code, examples and options.

From the onset, minimal Mathematica experience is needed to make use of the package.

It may be a helpful supplement in research and teaching where crystallography and X-ray diffraction is essential.

Although Mathematica is a proprietary software, all the code of the package is open source.

It may easily be extended to cover user-specific applications. 
References:

[1] Wolfram Research, Inc., (2018). Mathematica, Version 11.3. Champaign, IL, 2018.

[2] C. P. Brock, International Tables for Crystallography 5th Edition, 8 Volume Set, Wiley, 2016.

[3] Schoonjans, T., Brunetti, A., Golosio, B., del Rio, M. S., Solé, V. A., Ferrero, C. \& Vincze, L. (2011). Spectrochimica Acta Part B: Atomic Spectroscopy, 66(11), $776-784$.

Keywords: Mathematica, crystallography, computation

\section{MS41-04}

\section{Using DIALS with DUI}

Luis Fuentes-Montero', Gwyndaf Evans ${ }^{1}$

1. Diamond Light Source Ltd, Oxfordshire, United Kingdom email: luis.fuentes-montero@diamond.ac.uk

The DIALS User Interface (DUI) is a graphical user interface that is designed to make data processing with DIALS[1] more user-friendly and efficient. A key feature is a full history tree that keeps track of all steps of processing. Every position in the tree represents the execution of a DIALS command line with full record of the user-supplied parameters, and the results, which can be inspected with several visualisation tools. At any step, the user can either fork, proceed to the next command or navigate to another step without loss of information. This ability to keep track of different branches of data processing gives the user freedom to explore different hypotheses, such as the space group of the crystal.

The output and visualization tools in DUI are conveniently arranged in tabs that update their content when the user navigates the tree, allowing rapid comparisons to aid decision-making. Each tab provides a different view on the state at that position of the tree, from detailed log file output of the DIALS program and graphical reports to an interactive image viewer and a 3D reciprocal lattice viewer.

The image viewer has been redesigned for DUI to provide responsiveness alongside various image overlays that give information about the spots and experimental models. The reciprocal lattice viewer complements this by providing a view of reflection positions in reciprocal space. Together these tools provide a powerful means for identifying problematic cases that may not be so readily apparent from the graphs and statistics in the report and log files.

DUI is intended to be intuitive and immediately useful across a wide range of user expertise. Beginners learning about the integration process with DIALS will benefit from the ease-of-use of the GUI and the visualisation tools. Experts retain full control of the underlying DIALS programs and can use the history tree to test different ideas quickly.

In this presentation these features of DUI will be demonstrated with a rotation dataset from a macromolecular crystal. DUI is distributed to users as part of CCP4 7.0[2].

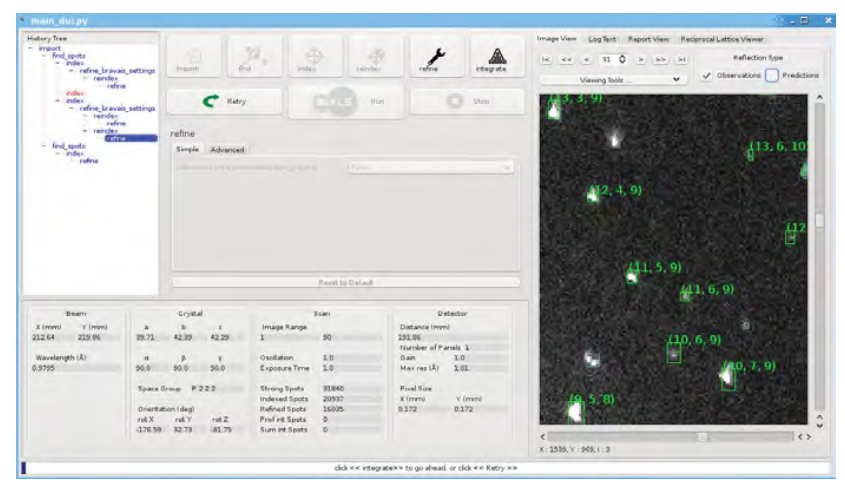

